\title{
Q1 Abdominal obesity as a mediator of the influence of physical activity on insulin resistance in Spanish adults 2 s
}

\author{
Q2 Antonio García-Hermoso a , Vicente Martínez-Vizcaíno ${ }^{\text {b,* }}$, Jose I. Recio Rodriguez ${ }^{c}$, Ana Díez-Fernández ${ }^{\text {, }}$, \\ Manuel A. Gómez Marcos ${ }^{c}$, Luis García Ortiz ${ }^{c}$, for the EVIDENT Group ${ }^{\mathrm{e}}$ \\ a Laboratorio de Ciencias de la Actividad Física, el Deporte y la Salud, Facultad de Ciencias Médicas, Universidad de Santiago de Chile, USACH, Chile \\ b Universidad de Castilla-La Mancha, Health and Social Research Center, Cuenca, Spain \\ c The Alamedilla Health Center, Castilla y León Health Service, SACYL, USAL, IBSAL, Salamanca, Spain \\ ${ }^{\mathrm{d}}$ Faculty of Occupational Therapy, Speech Therapy and Nursing, University of Castilla-La Mancha, Talavera de la Reina, Toledo, Spain \\ e EVIDENT Group, RedIAPP: Red de Investigación en Actividades Preventivas y Promoción de la Salud (Research Network on Preventive Activities and Health Promotion), Spain
}

\section{A B S T R A C T}

Available online $\mathrm{xxxx}$

\section{Keywords:}

Diabetes

Moderate-to-vigorous physical activity

Obesity

Waist circumference

Obesity

Cardiometabolic risk factors
Objective. The aim of the study was to analyze the relationship between moderate-to-vigorous physical 15 activity (MVPA) and insulin resistance (IR) in Spanish adults and to examine whether this relationship is 16 mediated by abdominal obesity (waist circumference - WC).

Methods. The cross-sectional study included 1162 healthy subjects belonging to the EVIDENT study (mean age $55.0 \pm 13.3$ years; $61.8 \%$ women) from six different Spanish provinces. Moderate-to-vigorous physical activ- 19 ity (MVPA) was measured objectively over 7 days using Actigraph accelerometers, collecting data in 60-second 20 epochs, and retaining respondents with $\geq 4$ valid days for the analysis. The homeostasis model of assessment 21 (HOMA-IR) was used to determine IR, and its individual components - fasting glucose and insulin - were deter- 22 mined using standard protocols. Linear regression models were fitted according to Baron and Kenny's procedures 23 for mediation analysis.

Results. Fasting insulin and HOMA-IR levels were significantly worse in adults who spent fewer minutes in 25 MVPA (first quartile $\leq 30.1$ and $22.7 \mathrm{~min}$ /day in men and women, respectively) after adjusting for age, sex, 26 smoking habits, drinking habits, accelerometer wear time, sedentary time, and Mediterranean diet adherence. 27 However, when WC was added to the ANCOVA models as a covariate, the effects disappeared. Mediation analysis 28 reported that WC acts as a full mediator in the relationship between MVPA and IR (HOMA-IR and fasting insulin). 29

Conclusion. These findings show that WC plays a pivotal role in the relationship between MVPA and IR, and 30 therefore highlights that decreasing abdominal obesity might be considered as an intermediate outcome for 31 evaluating interventions aimed at preventing diabetes mellitus.

\section{Introduction}

Physical activity is considered a protector factor against type 2 diabetes and cardiovascular risks (Jeon et al., 2007), and its benefits depend on the intensity, duration, and frequency of the activity performed (United States. Department of Health, 1996). International recommendations for adults include at least $150 \mathrm{~min} /$ week of moderate-tovigorous physical activity (MVPA) or 75 min of vigorous-intensity aerobic activity for at least 20 min 3 days a week (Haskell et al., 2007). Previous studies have shown an inverse association between MVPA with insulin resistance (IR) (Ekelund et al., 2009; Nelson et al., 2013) and abdominal obesity assessed by waist circumference (WC),

\footnotetext{
t) Trial registration: ClinicalTrials.gov identifier: NCT01083082.

* Corresponding author at: Universidad de Castilla-La Mancha, Edificio Melchor Cano, Centro de Estudios Socio-Sanitarios, Santa Teresa Jornet s/n, 16071 Cuenca, Spain.

E-mail address: Vicente.Martinez@uclm.es (V. Martínez-Vizcaíno).
}

(Henson et al., 2013; Scheers et al., 2013; Tremblay et al., 1990; Yang 56 et al., 2006), which has shown to be an appropriate proxy for abdominal 57 obesity (WHO, 2008). These are both related to cardiovascular risk 58 (CVR) (Haffner et al., 1998; Muniyappa et al., 2008), metabolic 59 syndrome (Zimmet et al., 2005) and type 2 diabetes (Wareham et al., 60 1999; Wilson et al., 2005). Therefore, increased MVPA levels both 61 decrease cardiovascular risk factors and improve the cardiometabolic 62 profile in the adult population.

A recent study with obese sedentary women has shown that WC 64 combined with cardiorespiratory fitness mediate the association 65 between MVPA and IR (Shalev-Goldman et al., 2013), while other 66 researchers have reported that this association remains unaffected 67 when adjusting for WC (Balkau et al., 2008; Ekelund et al., 2009). There- 68 fore, it seems necessary to clarify whether physical activity plays an 69 independent role as a protective factor of IR, or whether its influence 70 is mediated by changes in WC, given the close physiological link 71 between central obesity and IR (Carey et al., 1996). Mediation analysis 72 
is a statistical procedure that can be used to clarify the process underlying the relationship between two variables and the extent to which this relationship can be modified, mediated, or confounded by a third variable (Baron and Kenny, 1986). Thus, the aim of this study was twofold: i) to analyze the relationship between objectively measured MVPA and IR in Spanish adults and ii) to examine whether this relationship is mediated by abdominal obesity.

\section{Subjects and methods}

\section{Study design}

We conducted a cross-sectional analysis of the EVIDENT baseline study. Our study evaluated the association of lifestyles with the circadian pattern of blood pressure, arterial stiffness, and endothelial function in subjects with different levels of physical activity. The protocol of the EVIDENT study (NCT01083082) was published elsewhere (García-Ortiz et al., 2010).

\section{Subjects}

Subjects aged 20-80 years were selected through random sampling from the offices of general practitioners in six primary care centers from six different Spanish provinces (response rate ranged from 65\% to $85 \%$, depending on the center). The exclusion criteria have been published elsewhere (García-Ortiz et al., 2010). From the 1553 subjects included in the EVIDENT study, 391 were excluded due to lack of HOMA-IR (237) or accelerometer data (154), and therefore, 1162 patients were ultimately included in the analysis.

The Research Ethics Committee of Salamanca University Hospital (Spain) and the other centers involved approved this study. All participants gave written informed consent according to the general recommendations of the Declaration of Helsinki (World Medical Association, 2013).

\section{Measurements}

A detailed description was published elsewhere detailing how the measurements were collected (García-Ortiz et al., 2010). In short, we measured the following variables:

\section{Anthropometric measurements}

Body weight was determined on two occasions using a homologated electronic scale (Seca 770) (precision $\pm 0.1 \mathrm{~kg}$ ). Height, in turn, was measured with a portable system (Seca 222). Body mass index (BMI) and WC were also measured. WC was measured as follows: the upper border of the iliac crest was located, and the tape was wrapped around above this point, parallel to the floor, ensuring that it was adjusted without compressing the skin. The reading was taken at the end of a normal breath according to the recommendations of the 2007 SEEDO Conference (Salas-Salvado et al., 2007). In order to ensure the quality and reliability of the anthropometric measurements, the nurses responsible for the measurements were trained at the start of the project. We considered WC to be normal when the value was below $94 \mathrm{~cm}$ in men and $80 \mathrm{~cm}$ in women, according to the WHO cut-off points (WHO, 2008).

\section{Biochemical determinations}

Venous blood sampling was performed between 8:00 and 9:00 AM after the individuals had fasted (avoided smoking and the consumption of alcohol and caffeinated beverages for the previous $12 \mathrm{~h}$ ). Blood samples were collected in the respective health centers and were analyzed at the hospital of the city participating in external quality assurance programs of the Spanish Society of Clinical Chemistry and Molecular Pathology. Fasting plasma glucose was measured using standard enzymatic automated methods. The blood concentration of insulin was determined using a chemiluminescent microparticle immunoassay.
The insulin sensitivity was determined using the Homeostasis Model 130 Assessment Insulin Resistance (HOMA-IR) index with the following 131 formula: fasting glucose $(\mathrm{mmol} / \mathrm{l}) \times$ fasting insulin $(\mathrm{mU} / \mathrm{ml}) / 22.5 . \quad 132$

Physical activity and sedentary behavior

ActiGraph GT3X accelerometers (ActiGraph, Shalimar, FL, USA) 134 were used to assess physical activity and sedentary behavior. This previ- 135 ously validated accelerometer (Matthews et al., 2013; Melanson and 136 Freedson, 1995) measures acceleration in three individual orthogonal 137 planes (vertical, anteroposterior, and medio-lateral) and provides 138 activity counts as a composite vector magnitude of these three axes. 139

Participants were verbally instructed on how to use the accelerome- 140 ter, which was worn fastened with an elastic band to the right side of 141 the waist for 7 consecutive days. All subjects were instructed to wear 142 the accelerometer throughout the day from the time they woke up in 143 the morning until they went to bed at night, except for bathing and 144 performing activities in the water. Wear time was determined by 145 subtracting non-wear time from $24 \mathrm{~h}$. Non-wear time was defined as 146 an interval of at least 60 consecutive minutes of zero activity counts, 147 with allowance of up to 2 min of counts between 0 and 100 . The intensity 148 of PA was categorized according to the cut-off points Troiano et al. (2008) 149 proposed: sedentary (<100 counts/min), light (100-2019 counts/min), 150 moderate (2020-5998 counts/min), and vigorous ( $>5999$ counts/min). 151 MVPA time was calculated as the mean daily minutes $\geq 2020$ counts 152 per minute from all valid days.

Mediterranean diet adherence screener (MEDAS)

Adherence to the Mediterranean diet was assessed using the 155 validated 14-point MEDAS (Schroder et al., 2011), an adaptation of a 156 previously validated nine-item index. The MEDAS was developed by 157 the PREDIMED study group (Martinez-Gonzalez et al., 2004). The 158 questionnaire allows for easily estimating Mediterranean diet adher- 159 ence and may be useful in clinical settings. The 14-item version includes 160 12 questions about food consumption frequency and two questions 161 about food intake habit characteristics of the Spanish Mediterranean 162 diet. Further information about the diet assessment is available 163 elsewhere (Patino-Alonso et al., 2014).

Smoking and alcohol consumption

165

Smoking and alcohol consumption history was assessed through 166 questions on smoking status (current smokers or nonsmokers) and 167 alcohol consumption status (current consume alcohol or not consume). 168

\section{Statistical analysis}

169

The continuous variables were expressed as the mean \pm SD and 170 frequency distribution for categorical data. Statistical normality was 171 tested using both graphical (normal probability plot) and statistical 172 (Kolmogorov-Smirnov test) procedures. Because of their skewed distri- 173 bution, glucose, insulin and HOMA-IR were log-transformed (natural 174 logarithm) before being included in the models. To aid with interpreta- 175 tion, the data were back-transformed from the log scale for presentation 176 in the results.

177

ANCOVA models were estimated to test the differences in the mean 178 of fasting plasma glucose, insulin and HOMA-IR by categories of MVPA 179 (low $=$ quartile 1 ; medium = quartiles $2-3$; high = quartile 4), 180 controlling for age, smoking habits, drinking habits, accelerometer 181 wear time, total sedentary time, and Mediterranean diet adherence in 182 the first step (model 1), and including WC in the second step (model 183 2 ), by sex. Pairwise post hoc comparisons were examined using the 184 Bonferroni test.

To examine whether the association between physical activity and 186 IR parameters was mediated by WC, linear regression models were 187 fitted using bootstrapped mediation procedures included in the 188 PROCESS SPSS macro (Preacher and Hayes, 2008). The first equation 189 regressed the mediator (WC) on the independent variable (MVPA en- 190 tered as a continuous variable, $\min /$ day). The second equation regressed 191 
the dependent variable (fasting glucose, fasting plasma insulin and HOMA-IR) on the independent variable. The third equation regressed the dependent variable on both the independent and the mediator variables.

The following criteria were used to establish mediation: (1) the independent variable must be significantly related to the mediator, (2) the independent variable must be significantly related to the dependent variable, (3) the mediator must be significantly related to the dependent variable, and (4) the association between the independent and dependent variables must be attenuated when the mediator is included in the regression model (Baron and Kenny, 1986). In addition, we tested mediation using the steps Sobel (1982) outlined. First, we estimated the attenuation or indirect influence (i.e. the influence of the independent variable on the mediator from the first regression model multiplied by the influence of the mediator on the dependent variable obtained from the third regression model). Second, we divided the indirect influence by its standard error and performed a $\mathrm{Z}$ test under the null hypothesis that the indirect influence is equal to zero. This analysis was adjusted for age, sex, smoking habits, drinking habits and accelerometer wear time.

Statistical analyses were performed using IBM SPSS statistics software and the level of significance was set at $\alpha=0.05$.

\section{Results}

Data were obtained from 1162 subjects (mean age $55.0 \pm 13.3$ years), 718 (61.8\%) of whom were women. The anthropometric, IR parameters and accelerometer descriptive data of the sample are shown in Table 1 . Most subjects $(925 ; 80.0 \%)$ were non-smokers and did not consume alcohol (741;63.8\%), and on average, the men and women were $4.5 \mathrm{~cm}$ (for a total of $98.5 \mathrm{~cm}$ ) and $9.2 \mathrm{~cm}$ above WC cut-off point (for a total of $89.2 \mathrm{~cm}$ ), respectively. Mean fasting plasma glucose was higher in men than in women and the accelerometry data showed that men did more MVPA than women.

Mean differences in IR parameters according to MVPA categories are shown in Table 2. Men and women in the high MVPA categories had lower fasting plasma insulin and HOMA-IR values compared to those in the low categories after controlling for age, smoking habits, drinking 227 habits, accelerometer wear time, and sedentary time. However, when 228 we included additional adjustment for WC (model 2), the differences 229 disappeared.

In both sexes, WC acted as a full mediator of the relationship be- 231 tween MVPA and fasting plasma insulin (Fig. 1A). Similar results were 232 obtained when HOMA-IR was the dependent variable in the mediation 233 model (Fig. 1B). The estimated percentage of total influence mediated 234 by WC was $12.3 \%$ in men $(z=-2.03, p=0.042)$ and $20.6 \%$ in 235 women $(z=-3.07, p=0.002)$ for fasting plasma insulin, and $14.1 \% 236$ $(z=-2.06 ; p=0.039)$ in men and $23.2 \%(z=-3.13, p=0.002)$ in 237 women for HOMA-IR. Finally, the criteria necessary for mediation 238 analysis were not achieved for fasting glucose levels (data not reported). 239

\section{Discussion}

Although a growing number of studies have examined the associa- 241 tion between physical activity and IR, none of them have evaluated 242 the mediation role of excess weight in this relationship in healthy 243 adults. Our results show that MVPA was negatively associated with IR 244 parameters, even after adjusting for sociodemographic and lifestyle po- 245 tential confounders, but this association disappeared when additional 246 adjustments for WC were included. Moreover, the mediation analysis 247 disclosed that the influence of MVPA on IR was mediated by abdominal 248 obesity, as measured by WC.

There is consistent evidence regarding the beneficial influence of 250 MVPA on insulin sensitivity and glycemic control in adults, independent 251 of weight change (Ekelund et al., 2007; Ivy, 1997), as well as on abdom- 252 inal obesity, as measured by WC (Staiano et al., 2012; Stamatakis et al., 253 2009). Previous studies have shown that subjects with higher values of 254 WC presented worse IR profiles, and those who were physically active 255 presented lower IR values in the same WC category compared to their 256 sedentary peers (Ekelund et al., 2007; Healy et al., 2006; Nelson et al., 257 2013; Weinstein et al., 2004).

Some other studies have described that there is a relationship be- 259 tween PA and IR, independent of WC (Balkau et al., 2008; Ekelund 260 et al., 2009), while others have reported that the amount of physical 261

Table 1

Demographic and clinical characteristics of patients, by sex.

\begin{tabular}{|c|c|c|c|c|}
\hline & Total $(n=1162)$ & $\begin{array}{l}\operatorname{Men}^{\mathrm{a}} \\
(n=444)\end{array}$ & Women $^{\mathrm{a}}(n=718)$ & $p$ \\
\hline Age (years) & $55.0(13.3)$ & $57.3(14.5)$ & $53.5(16.0)$ & $<0.001$ \\
\hline \multicolumn{5}{|l|}{ Smoking status, $n(\%)$} \\
\hline Yes & $237(20.0)$ & $83(18.6)$ & $154(21.4)$ & \multirow[t]{2}{*}{0.431} \\
\hline No or past & $925(80.0)$ & $361(81.4)$ & $564(78.6)$ & \\
\hline \multicolumn{5}{|l|}{ Alcohol status, $n(\%)$} \\
\hline Yes & $421(36.2)$ & $116(26.1)$ & $305(42.5)$ & \multirow[t]{2}{*}{$<0.001$} \\
\hline No or past & $741(63.8)$ & $328(73.9)$ & $413(57.5)$ & \\
\hline Weight (kg) & $72.8(17.0)$ & $81.8(12.7)$ & $67.2(13.4)$ & $<0.001$ \\
\hline Height $(\mathrm{cm})$ & $163.2(10.2)$ & $170.8(8.4)$ & $158.6(8.1)$ & $<0.001$ \\
\hline Body mass index $\left(\mathrm{kg} / \mathrm{m}^{2}\right)$ & $27.3(3.4)$ & $28.0(4.2)$ & $26.8(5.4)$ & $<0.001$ \\
\hline Waist circumference $(\mathrm{cm})$ & $92.8(13.6)$ & $98.5(10.5)$ & $89.2(13.4)$ & $<0.001$ \\
\hline \multicolumn{5}{|l|}{ Obesity $^{\mathrm{b}} n(\%)$} \\
\hline Yes & $863(74.3)$ & $291(65.5)$ & $572(79.7)$ & \multirow[t]{2}{*}{$<0.001$} \\
\hline No & $299(25.7)$ & $153(34.5)$ & $146(20.3)$ & \\
\hline Fasting plasma glucose $(\mathrm{mmol} / \mathrm{L})$ & $5.08(1.4)$ & $5.26(1.3)$ & $4.96(1.1)$ & $<0.001$ \\
\hline Fasting plasma insulin $(\mu \mathrm{mol} / \mathrm{L})$ & $7.48(6.5)$ & $7.78(5.9)$ & $7.30(6.7)$ & 0.228 \\
\hline HOMA-IR & $1.75(1.7)$ & $1.88(1.7)$ & $1.68(1.9)$ & 0.068 \\
\hline Accelerometer wear time (min/day) & $931.2(218.0)$ & $941.6(231.0)$ & $924.8(210.6)$ & 0.239 \\
\hline MVPA (min/day) & $46.5(28.6)$ & $53.8(31.3)$ & $42.0(25.9)$ & $<0.001$ \\
\hline Meet recommendations for $\mathrm{MVPA}^{\mathrm{c}} n(\%)$ & $527(45.3)$ & $233(52.5)$ & $294(41.0)$ & $<0.001$ \\
\hline VPA (min/day) & $1.22(5.4)$ & $2.0(7.8)$ & $0.7(3.0)$ & $<0.001$ \\
\hline Sedentary time (min/day) & $580.3(175.6)$ & $601.7(186.6)$ & $567.2(167.4)$ & 0.003 \\
\hline Healthy diet score $(0-14)$ & $7.4(1.8)$ & $7.4(1.9)$ & $7.5(1.7)$ & 0.386 \\
\hline Mediterranean diet adherence, ${ }^{\mathrm{b}} n(\%)$ & $337(29.0)$ & $120(27.1)$ & $217(30.2)$ & 0.304 \\
\hline
\end{tabular}

MVPA, moderate-vigorous physical activity; VPA, vigorous physical activity.

a Values are means $\pm \mathrm{SD}$, except for categorical data $(n(\%))$.

b Cut-off for obesity were waist circumference greater than $94 \mathrm{~cm}$ for men and $80 \mathrm{~cm}$ for women (WHO, 2008).

c $150 \mathrm{~min} /$ week of MVPA (Haskell et al., 2007). 
Table 2

ANCOVA models testing differences in fasting plasma glucose, insulin, and HOMA-IR by MVPA categories, adjusting for potential confounders, by sex.

\begin{tabular}{|c|c|c|c|c|c|c|c|}
\hline & & \multicolumn{2}{|c|}{$\begin{array}{l}\text { Fasting plasma glucose }{ }^{\mathrm{a}} \\
(\mathrm{mmol} / \mathrm{L})\end{array}$} & \multicolumn{2}{|c|}{$\begin{array}{l}\text { Fasting plasma insulin }{ }^{\mathrm{a}} \\
(\mu \mathrm{mol} / \mathrm{L})\end{array}$} & \multicolumn{2}{|l|}{ HOMA-IR ${ }^{\mathrm{a}}$} \\
\hline & & Men & Women & Men & Women & Men & Women \\
\hline \multirow{5}{*}{ Model 1} & Low (L) & $5.44 \pm 1.18$ & $5.20 \pm 1.44$ & $9.62 \pm 6.30$ & $8.97 \pm 6.86$ & $2.46 \pm 2.14$ & $2.21 \pm 2.24$ \\
\hline & Medium (M) & $5.41 \pm 1.48$ & $4.92 \pm 0.79$ & $8.41 \pm 4.09$ & $7.16 \pm 5.14$ & $2.06 \pm 1.09$ & $1.58 \pm 1.13$ \\
\hline & $\operatorname{High}(\mathrm{H})$ & $5.15 \pm 0.99$ & $4.87 \pm 0.76$ & $7.24 \pm 4.47$ & $6.64 \pm 5.13$ & $1.69 \pm 1.13$ & $1.47 \pm 1.18$ \\
\hline & $p$ & 0.139 & 0.223 & 0.030 & 0.001 & 0.004 & 0.002 \\
\hline & Post-hoc tests ${ }^{b}$ & ns & ns & $\mathrm{L}>\mathrm{H}$ & $\mathrm{L}>\mathrm{H}$ & $\mathrm{L}>\mathrm{H}$ & $\mathrm{L}>\mathrm{H}$ \\
\hline \multirow[t]{2}{*}{ Model 2} & $p$ & 0.134 & 0.719 & 0.213 & 0.114 & 0.201 & 0.191 \\
\hline & Post-hoc tests & ns & ns & ns & ns & ns & ns \\
\hline
\end{tabular}

\section{Values are means \pm SD}

Abbreviations: HOMA-IR, homeostatic model assessment of insulin resistance; MVPA, moderate-vigorous physical activity; ns, non-significant.

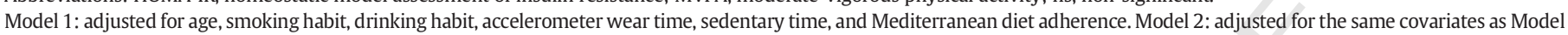
1 and waist circumference.

a Log transformed for comparing means.

b Bonferroni pairwise comparisons.

activity was not associated with IR after controlling for WC (ShalevGoldman et al., 2013). Our data show that participants in the low MVPA category had higher fasting plasma insulin and HOMA-IR levels than did those in the higher category, but after adjusting for WC, the relationship between MVPA and IR disappeared. Likewise, Healy et al. (2008) found that the inverse association of MVPA with cardiometabolic risk factors did not remain significant when WC was included as a confounder, except for triglyceride levels.

Discrepancies between studies can be explained by differences in the populations studied (e.g. health risk status, weight status, ethnicity and age), in the methods for physical activity assessment (e.g. calibrated heart rate monitoring or accelerometers), and in the control of confounders (e.g. sedentary time, light intensity physical activity). The main difference between our results and those presented by ShalevGoldman et al. (2013) is that we calculated the magnitude of WC as a single mediator, finding that it affects individuals in the causal pathway between MVPA and IR.

This evidence allows us to conclude that active people are more likely to present a healthy IR and cardiometabolic profile, and thus being physically active may help to prevent metabolic syndrome and type 2 diabetes. It also confirms the potential mediation role of abdominal obesity, as other authors have suggested (Alberti et al., 2005). Therefore, our cross-sectional study suggests that the influence of physical activity on improving IR is substantially greater when accom- 285 panied by a decrease in WC.

Furthermore, our non-significant findings for fasting plasma glucose 287 are in agreement with the results from most previous studies (Henson 288 et al., 2013; Scheers et al., 2013; Shalev-Goldman et al., 2013), and em- 289 phasizing that fasting plasma glucose might not be sensitive to changes 290 in physical activity, and two-hour plasma glucose appears to be a more 291 appropriate biomarker for use as an outcome measure in interventions 292 that are based on physical activity (Healy et al., 2008).

Mediation analysis

Our data, by using mediation analysis, reveal that abdominal obesity 295 has a powerful influence on the relationship between MVPA and both 296 IR and fasting plasma insulin in adults. Visceral or central obesity in- 297 creases the flux of free fatty acids to the liver, and an important role in 298 the genesis of IR of these free fatty acids has been described (Petersen 299 et al., 2004). Inflammation has also been reported as an alternative candi- 300 date for the common link between central obesity and IR (Ghanim et al., 301 2004). Finally, increased abdominal obesity has been associated not only 302 with impaired insulin sensitivity but also with reduced glycogen synthesis 303 (non-oxidative glucose disposal) and a reduced responsiveness of muscle 304

\section{B}
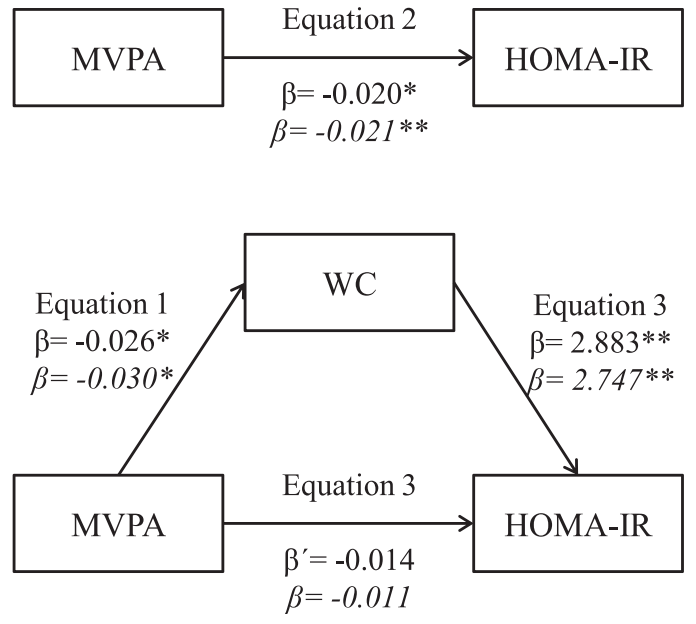

Sobel test $=-2.03 * ;-3.12 * *$

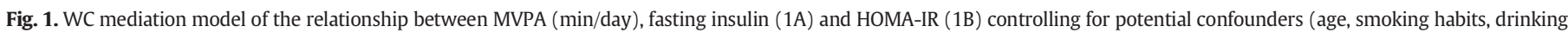
habits, accelerometer wear time, sedentary time, and Mediterranean diet adherence), by sex. Data in roman type refer to men. Data in italics refer to women. ${ }^{*} p \leq 0.05 ;{ }^{* *} p \leq 0.001$. 
glycogen synthase (Carey et al., 1996), all of which are related to central obesity and an increased risk of IR.

These physiological pathways support the hypothesis that programs aimed at increasing physical activity could diminish IR though reductions of abdominal obesity, thus you can expect a weak impact on the insulin metabolism of those physical activity programs that, while improving other cardiometabolic factors, fail to diminish adiposity. Besides, the relationships analyzed here are probably related to more than a single mediator variable (e.g. BMI, accumulated daily activity, sedentary time); future studies using structural equations or complex mediator model procedures might be useful in clarifying more specifically the potential mediator or confounder role of each factor.

Finally, it might be also interesting to address how these results may affect those subjects defined as metabolically healthy obese (MHO) vs. metabolically unhealthy obese (MUHO) or non-obese. Previous studies have found that MHO subjects had intermediate levels of WC (Després, 2012), a more favorable fat distribution (lower visceral fat and greater thigh subcutaneous fat), normal levels of C-reactive protein (Phillips, 2013) and higher PA levels (Wildman et al., 2008) compared to MUHO and non-obese subjects. A recent study in a Spanish population (Martínez-Larrad et al., 2014) reported that the prevalence of MHO subjects was $4.78 \%$ according to Wildman modified criteria (Ascaso et al., 2001). Thus, because of this relatively low prevalence, our coefficients' estimations will probably remain very similar if $\mathrm{MHO}$ individuals are removed from the analyses, but our mediation results should be applied with caution in these subjects, since indirect coefficients through WC might be substantially different in this MHO phenotype.

This study has several limitations. First, the cross-sectional design prevents us from establishing a causal relationship. For example, we cannot establish that abdominal obesity predicts greater MVPA, since it seems reasonable to think that many adults begin physical activity due to an awareness of excess weight. Second, we did not use an intravenous glucose tolerance test or hyperinsulinemic-euglycemic clamp technique, both considered better measures of IR (DeFronzo et al., 1979), but instead, we used HOMA-IR, which is probably the most appropriate method for measuring IR in large epidemiological studies. Third, PA was assessed using an accelerometer during the same week, and the fact that the subjects were evaluated with the device might have influenced their activity levels that week. However, without any activity goals communicated, the possible effect seems to be negligible. Fourth, we studied subjects that were randomly selected from outpatient clinics in different regions of Spain; however, the subjects may not be representative of the general Spanish population, but rather, of people that attend primary care centers. We must also take into consideration the limited generalizability of the study because the participants were people who met the rigorous inclusion criteria of the parent trial. The generalizability is also limited because of the impossibility of adjusting for all potential confounders, and this causes the residual confounding to tease out independent associations. Finally, because of the limited amount of time spent in vigorous physical activity in our sample ( 8 out of 10 participants accumulated no vigorous physical activity), statistics that are similar to those reported by other authors (Ekelund et al., 2009), it was not feasible to examine the relative importance of vigorous physical activity on IR. However, we found a relationship between insulin, IR and vigorous physical activity in men, independent of WC, suggesting that obesity mechanisms may not be so relevant (Janssen and Ross, 2012) in this population.

\section{Conclusion}

Our findings are important from a clinical and public health pointof-view because they disclose that abdominal obesity plays a critical role in the relationship between MVPA and IR, and they alert us that physical activity only produces benefits in IR if the activity is not accompanied by compensatory behaviors that might mitigate its influence on WC (e.g. less sedentary time or nutritional changes).

\section{References}

Alberti, K.G.M., Zimmet, P., Shaw, J., 2005. The metabolic syndrome-a new worldwide 370 definition. Lancet 366, 1059-1062.

Ascaso, J.F., Romero, P., Real, J.T., Priego, A., Valdecabres, C., Carmena, R., 2001. Insulin 372 resistance quantification by fasting insulin plasma values and HOMA index in a 373 non-diabetic population. Med. Clín. 117, 530-533.

Balkau, B., Mhamdi, L., Oppert, J.-M., Nolan, J., Golay, A., Porcellati, F., Laakso, M., 375 Ferrannini, E., 2008. Physical activity and insulin sensitivity the RISC study. Diabetes 376 57, 2613-2618.

Baron, R.M., Kenny, D.A., 1986. The moderator-mediator variable distinction in social 378 psychological research: conceptual, strategic, and statistical considerations. J. Pers. 379 Soc. Psychol. 51, 1173

Carey, D.G., Jenkins, A.B., Campbell, L.V., Freund, J., Chisholm, D.J., 1996. Abdominal fat and 381 insulin resistance in normal and overweight women: direct measurements reveal a 382 strong relationship in subjects at both low and high risk of NIDDM. Diabetes 45, 383 633-638.

DeFronzo, R.A., Tobin, J.D., Andres, R., 1979. Glucose clamp technique: a method for 385 quantifying insulin secretion and resistance. Am. J. Physiol. Gastrointest. Liver Physiol. 386 237, G214-G223.

Després, J.-P., 2012. What is "metabolically healthy obesity"?: from epidemiology to 388 pathophysiological insights. J. Clin. Endocrinol. Metab. 97, 2283-2285. 389

Ekelund, U., Franks, P.W., Sharp, S., Brage, S., Wareham, N.J., 2007. Increase in physical 390 activity energy expenditure is associated with reduced metabolic risk independent 391 of change in fatness and fitness. Diabetes Care 30, 2101-2106.

Ekelund, U., Brage, S., Griffin, S.J., Wareham, N.J., 2009. Objectively measured moderate- 393 and vigorous-intensity physical activity but not sedentary time predicts insulin 394 resistance in high-risk individuals. Diabetes Care 32, 1081-1086. artiz, L., Recio-Rodríguez, J.I., Martín-Cantera, C., Cabrejas-Sánchez, A., Gómez- 396 Arranz, A., González-Viejo, N., Nicolás, E.I., Patino-Alonso, M.C., Gómez-Marcos, 397 M.A., 2010. Physical exercise, fitness and dietary pattern and their relationship with 398 circadian blood pressure pattern, augmentation index and endothelial dysfunction 399 biological markers: EVIDENT study protocol. BMC Public Health 10, 233.

Ghanim, H., Aljada, A., Hofmeyer, D., Syed, T., Mohanty, P., Dandona, P., 2004. Circulating 401 mononuclear cells in the obese are in a proinflammatory state. Circulation 110, 402 $1564-1571$.

Haffner, S.M. Lehto, S, Rönnemaa, T, Pyörälä, K, Laakso, M. 1998. Mortality from 404 coronary heart disease in subjects with type 2 diabetes and in nondiabetic subjects 405 with and without prior myocardial infarction. N. Engl. J. Med. 339, 229-234.

Haskell, W.L., Lee, I.M., Pate, R. R., Powell, K.E. Blair, S.N., Franklin, B.A., Macera, C.A., Heath, 407 G.W., Thompson, P.D., et al., 2007. Physical activity and public health: updated 408 recommendation for adults from the American College of Sports Medicine and the 409 American Heart Association. Med. Sci. Sports Exerc. 39, 1423-1434.

Healy, G.N., Dunstan, D.W. Shaw, J.E., Zimmet, P.Z., Owen, N., 2006. Beneficial associations 411 of physical activity with 2-h but not fasting blood glucose in Australian adults: the 412 AusDiab study. Diabetes Care 29, 2598-2604.

Healy, G.N., Wijndaele, K., Dunstan, D.W., Shaw, J.E., Salmon, J., Zimmet, P.Z., Owen, N., 414 2008. Objectively measured sedentary time, physical activity, and metabolic risk 415 the Australian Diabetes, Obesity and Lifestyle study (AusDiab). Diabetes Care 31, 416 369-371.

Henson, J., Yates, T., Biddle, S., Edwardson, C., Khunti, K., Wilmot, E., Gray, L, Gorely, T 418 Nimmo, M., et al., 2013. Associations of objectively measured sedentary behaviour 419 and physical activity with markers of cardiometabolic health. Diabetologia 1-9. 420

Ivy, J.L., 1997. Role of exercise training in the prevention and treatment of insulin 421 resistance and non-insulin-dependent diabetes mellitus. Sports Med. 24, 321-336. 422

Janssen, I., Ross, R., 2012. Vigorous intensity physical activity is related to the metabolic 423 syndrome independent of the physical activity dose. Int. J. Epidemiol. 41, 1132-1140. 424

Jeon, C.Y., Lokken, R.P., Hu, F.B., Van Dam, R.M., 2007. Physical activity of moderate inten- 425 sity and risk of type 2 diabetes: a systematic review. Diabetes Care 30, 744-752. 426

Martinez-Gonzalez, M.A., Fernandez-Jarne, E., Serrano-Martinez, M., Wright, M., Gomez- 427 Gracia, E., 2004. Development of a short dietary intake questionnaire for the 428 quantitative estimation of adherence to a cardioprotective Mediterranean diet. Eur. 429 J. Clin. Nutr. 58, 1550-1552.

Martínez-Larrad, M.T., Anchuelo, A.C., Del Prado, N., Rueda, J.M.I., Gabriel, R., Serrano-Ríos, 431 M., 2014. Profile of individuals who are metabolically healthy obese using different 432 definition criteria. A population-based analysis in the Spanish population. PLoS One 433 9, e106641.

Matthews, C.E., Keadle, S.K., Sampson, J., Lyden, K., Bowles, H.R., Moore, S.C., Libertine, A., 435 Freedson, P. . Fowke, J.H. 2013. Validation of a previous-day recall measure of active 436 and sedentary behaviors. Med. Sci. Sports Exerc. 45 (8), 1629-1638. 437 Melanson, E., Freedson, P.S., 1995. Validity of the Computer Science and Applications, Inc. 438 (CSA) activity monitor. Med. Sci. Sports Exerc. 27, 934-940.

Muniyappa, R., Lee, S., Chen, H., Quon, MJ. 2008. Current approa sensitivity and resistance in vivo: advantages, limitations, and appropriate usage. Am. 441 J. Physiol. Endocrinol. Metab. 294, E15-E26.

Nelson, RK, Horowitz, JF., Holleman, R.G. Swartz, AM, Strath, S. Kriska, A.M., Richardson, C.R., 2013. Daily physical activity predicts degree of insulin resistance: 444 a cross-sectional observational study using the 2003-2004 National Health and 445 Nutrition Examination Survey. Int. J. Behav. Nutr. Phys. Act. 10, 10.1186.

Patino-Alonso, M.C., Recio-Rodríguez, J.I., Belio, J.F.M., Colominas-Garrido, R., 447 Lema-Bartolomé, J., Arranz, A.G., Agudo-Conde, C., Gomez-Marcos, M.A., García-Ortiz, 448 L., 2014. Factors associated with adherence to the Mediterranean diet in the adult 449 population. J. Acad. Nutr. Diet. 114, 583-589.

Petersen, K.F., Dufour, S., Befroy, D., Garcia, R., Shulman, G.I., 2004. Impaired mitochondrial 451 activity in the insulin-resistant offspring of patients with type 2 diabetes. N. Engl. 452 J. Med. 350, 664-671. 
Phillips, C.M., 2013. Metabolically healthy obesity: definitions, determinants and clinical implications. Rev. Endocr. Metab. Disord. 14, 219-227.

Preacher, K.J., Hayes, A.F., 2008. Asymptotic and resampling strategies for assessing and comparing indirect effects in multiple mediator models. Behav. Res. Methods 40, 879-891.

Salas-Salvado, J., Rubio, M.A., Barbany, M., Moreno, B., Grupo, C.d.I.S., 2007. SEEDO 2007 Consensus for the evaluation of overweight and obesity and the establishment of therapeutic intervention criteria. Med. Clín. 128, 184.

Scheers, T., Philippaerts, R., Lefevre, J., 2013. SenseWear-determined physical activity and sedentary behavior and metabolic syndrome. Med. Sci. Sports Exerc. 45, 481-489.

Schroder, H., Fito, M., Estruch, R., Martinez-Gonzalez, M.A., Corella, D., Salas-Salvado, J., Lamuela-Raventos, R., Ros, E., Salaverria, I., Fiol, M., Lapetra, J., Vinyoles, E., GómezGracia, E., Lahoz, C., Serra-Majem, L., Pintó, X., Ruiz-Gutierrez, V., Covas, M.I., 2011. A short screener is valid for assessing Mediterranean diet adherence among older Spanish men and women. J. Nutr. 141, 1140-1145.

Shalev-Goldman, E., McGuire, K.A., Ross, R., 2013. Waist circumference and cardiorespiratory fitness are independently associated with glucose tolerance and insulin resistance in obese women. Appl. Physiol. Nutr. Metab. 39, 358-362.

Sobel, M.E., 1982. Asymptotic confidence intervals for indirect effects in structural equation models. Sociol. Methodol. 13, 290-312.

Staiano, A.E., Reeder, B.A., Elliott, S., Joffres, M.R., Pahwa, P., Kirkland, S.A., Paradis, G., Katzmarzyk, P.T., 2012. Physical activity level, waist circumference, and mortality. Appl. Physiol. Nutr. Metab. 37, 1008-1013.

Stamatakis, E., Hirani, V., Rennie, K., 2009. Moderate-to-vigorous physical activity and sedentary behaviours in relation to body mass index-defined and waist circumference-defined obesity. BJN 101, 765-773.

Tremblay, A., Després, J.-P., Leblanc, C., Craig, C.L., Ferris, B., Stephens, T., Bouchard, C., 1990. Effect of intensity of physical activity on body fatness and fat distribution. Am. J. Clin. Nutr. 51, 153-157.
Troiano, R.P., Berrigan, D., Dodd, K.W., Mâsse, L.C., Tilert, T., McDowell, M., 2008. Physical 483 activity in the United States measured by accelerometer. Med. Sci. Sports Exerc. 40, 484 $181-188$.

United States, Department of Health, 1996. Physical Activity and Health: A Report of the 486 Surgeon General. DIANE Publishing.

Wareham, N.J., Byrne, C.D., Williams, R., Day, N.E., Hales, C.N., 1999. Fasting proinsulin 488 concentrations predict the development of type 2 diabetes. Diabetes Care 22, 489 262-270.

Weinstein, A.R., Sesso, H.D., Lee, I.M., Cook, N.R., Manson, J.E., Buring, J.E., Gaziano, J.M., 491 2004. Relationship of physical activity vs body mass index with type 2 diabetes in 492 women. JAMA 292, 1188-1194.

Wildman, R.P., Muntner, P., Reynolds, K., McGinn, A.P., Rajpathak, S., Wylie-Rosett, J., 494 Sowers, M.R., 2008. The obese without cardiometabolic risk factor clustering and 495 the normal weight with cardiometabolic risk factor clustering: prevalence and corre- 496 lates of 2 phenotypes among the US population (NHANES 1999-2004). Arch. Intern. 497 Med. 168, 1617-1624.

Wilson, P.W., D'Agostino, R.B., Parise, H., Sullivan, L., Meigs, J.B., 2005. Metabolic syndrome 499 as a precursor of cardiovascular disease and type 2 diabetes mellitus. Circulation 112, 500 3066-3072.

World Health Organization, 2008. Waist Circumference and Waist-Hip Ratio Report of a 502 WHO Expert Consultation (Geneva, 8-11 December 2008). 503

World Medical Association, 2013. World Medical Association Declaration of Helsinki: eth- 504 ical principles for medical research involving human subjects. JAMA 310, 2191-2194. 505

Yang, X., Telama, R., Viikari, J., Raitakari, O.T., 2006. Risk of obesity in relation to physical 506 activity tracking from youth to adulthood. Med. Sci. Sports Exerc. 38, 919-925. 507

Zimmet, P., Magliano, D., Matsuzawa, Y., Alberti, G., Shaw, J., 2005. The metabolic 508 syndrome: a global public health problem and a new definition. J. Atheroscler. 509 Thromb. 12 (6), 295-300. 